\title{
Carbons from Biomasic Waste and Their Applications
}

\author{
Jorge Bedia *(i) and Carolina Belver *(1) \\ Departamento de Ingeniería Química, Facultad de Ciencias, Universidad Autónoma de Madrid, \\ Campus Cantoblanco, E-28049 Madrid, Spain \\ * Correspondence: jorge.bedia@uam.es (J.B.); carolina.belver@uam.es (C.B.); \\ Tel.: +34-91-497-2911 (J.B.); +34-91-497-8473 (C.B.)
}

Received: 18 September 2019; Accepted: 23 September 2019; Published: 26 September 2019

Carbon-based materials, such as chars, activated carbons, one-dimensional carbon nanotubes, and two-dimensional graphene nanosheets, have shown great potential for a wide variety of applications. These materials have innumerable advantages, such as low-cost, tunable porosity, variety of morphology, ease of processability, and possible modification of surface properties by heteroatom doping. Many of the carbon-based compounds are currently obtained from fossil fuels. However, the unstable cost and supply of fossil fuels have focused attention on the synthesis of carbon materials from biomass feedstocks. Biomass is an abundant renewable resource that has received growing attention as a raw material for production of advanced carbon-based materials because of the added value of the final products and the environmentally friendly characteristics of the synthesis processes. In this respect, various routes have been used to synthesize diverse structures of biomass-based carbon materials, including carbonization, hydrocarbonization or activation. Taking this into account, the main aim of this Special Issue of $C$ - Journal of Carbon Research—is to present the most relevant and recent insights in the field of synthesis of biomass-derived carbons for sustainable applications, from adsorption to energy storage applications.

To this end, Bernal et al. [1] analyzed the adsorption of different pharmaceuticals (salicylic acid, acetaminophen, and methylparaben) on activated carbons from coconut shell. The carbons were treated by an $\mathrm{HNO}_{3}$ oxidation or heat treatment at different temperatures to generate different surface chemistries. The effect of the physicochemical changes of the adsorbent in the adsorption capacity of the compounds was determined from the equilibrium data (adsorption isotherms), and the adsorbate-adsorbent affinity was evaluated by immersion enthalpy in the studied compound solutions. The study concluded that the activated carbons with highest content of acidic groups had lower adsorption capacities of the pharmaceutical compounds, while those activated carbons with amphoteric characteristics showed higher adsorption capacities. This behavior was ascribed to the increased affinity due to the presence of polar groups and corroborated by the high immersion enthalpy value in water. In another study, Contescu et al. [2] studied the potential conversion of biomass-derived chars into higher value activated carbons by a low-cost process. Six chars derived from lignocellulosic precursors were activated with $\mathrm{CO}_{2}$ and further characterized. In similar activation conditions, the surface area of the activated carbons correlates with the activation energy of the oxidation reaction by $\mathrm{CO}_{2}$, which in turn varies inversely with the carbon yield after thermolysis in nitrogen at $1000^{\circ} \mathrm{C}$. Since lignin is the most thermally-stable component of lignocellulosic biomass, these results demonstrate, indirectly, that robust, lignin-rich vegetal precursors are to be preferred to produce higher quality activated carbons. The chars derived from white pine (Pinus strobus) and chestnut oak (Quercus prinus) were converted to activated carbons with the highest surface area $\left(900-1100 \mathrm{~m}^{2} / \mathrm{g}\right.$ ) and largest mesopores volume $\left(0.85-1.06 \mathrm{~cm}^{3} / \mathrm{g}\right)$. These activated carbons have properties similar to those of commercially-available activated carbons used successfully for removal of pollutants from aqueous solutions. The authors concluded that still more work is needed to understand the relationship between the properties of 
initial biomaterials, charred derivatives, and the activated carbons obtained by physical activation. The properties of activated carbons synthesized from biomass (spruce and birch chips) in a thermal process, either in a direct carbonization-activation process or by first carbonizing the biomass and later activating the bio-chars into activated carbons, was studied by Bergna et al. [3]. Slightly different activated carbons were produced depending on whether a one-stage or a two-stage carbonization and activation process was used. Those produced in the one-stage process had higher specific surface areas than those synthesized in a two-stage process. There was no significant difference in total carbon content in general between the one-stage and two-stage processes. Hossain et al. [4] reported the preparation of mesoporous activated carbon with high surface area from hemp bast fiber using a hydrothermal treatment followed by an activation step using $\mathrm{KOH}$ and $\mathrm{NaOH}$ at different mass ratios. This procedure enhanced the mesoporosity and yielded high surface area activated carbons. The procedure described in this study is an environmentally friendly scalable route for the mass production of activated carbon using hemp fiber.

In addition to the research papers, this Special Issue also include two extensive review studies. Liu et al. [5] reviewed the synthesis of biomass-derived carbon in energy storage application. Biomass carbons have attracted increasing research attention due to the ease of fabrication, cost-effectiveness, and sustainability. Compared to the artificial nanostructured carbons, such as fullerene, carbon nanotube and graphene, the biomass-derived carbons may obtain superior capacitance, rate performance and stability in supercapacitor applications as a result of their intrinsic nanoporous and hierarchical structures. However, challenges remain in processing techniques to obtain biomass-derived carbons with high carbon yield, high energy density, and controllable graphitic microstructures, which may require a clear understanding over the chemical and elemental compositions, and the intrinsic microstructural characteristics of the biological precursors. The review presents comprehensive analyses over the impacts of the chemical and elemental compositions of the precursors on the carbon yield of the biomass, as well as the mechanism of chemical activation on the nanoporous structure development of the biomass-derived carbons. The structure-property relationship and functional performance of various biomass-derived carbons for supercapacitor applications are also discussed in detail and compared. Finally, useful insights are also provided for the improvements of biomass-derived carbons in supercapacitor applications. Finally, our own research group analyzed the synthesis and characterization of biomass-derived carbons for adsorption of emerging contaminants from water [6]. This review identifies the different types of emerging contaminants more often found in water streams, including a brief reference to the available technologies for their removal. It also describes the biomass sources that could be used for the synthesis of biochars and activated carbons, the characterization techniques available to understand the properties of the adsorbents, and the different approaches that can be followed to learn about the adsorption processes. The work also includes a review of the literature studies focused on the adsorption of emerging contaminants on biochars and activated carbons synthesized from biomass precursors.

After perusing the contents of this Special Issue, it is clear that the preparation of carbon materials from biomass waste can be considered a very promising research area, with a high amount of applications in diverse research fields. As Guest Editors of this Special Issue, we would like to thank all the authors for their excellent studies, and we hope that the readers find interesting and novel the results and conclusions included in it.

Conflicts of Interest: The authors declare no conflict of interest.

\section{References}

1. Bernal, V.; Giraldo, L.; Moreno-Piraján, J.C. Physicochemical Properties of Activated Carbon: Their Effect on the Adsorption of Pharmaceutical Compounds and Adsorbate-Adsorbent Interactions. C 2018, 4, 62. [CrossRef] 
2. Contescu, C.I.; Adhikari, S.P.; Gallego, N.C.; Evans, N.D.; Biss, B.E. Activated Carbons Derived from High-Temperature Pyrolysis of Lignocellulosic Biomass. C 2018, 4, 51. [CrossRef]

3. Bergna, D.; Varila, T.; Romar, H.; Lassi, U. Comparison of the Properties of Activated Carbons Produced in One-Stage and Two-Stage Processes. C 2018, 4, 41. [CrossRef]

4. Hossain, M.Z.; Wu, W.; Xu, W.Z.; Chowdhury, M.B.I.; Jhawar, A.K.; Machin, D.; Charpentier, P.A. High-Surface-Area Mesoporous Activated Carbon from Hemp Bast Fiber Using Hydrothermal Processing. C 2018, 4, 38. [CrossRef]

5. Liu, Y.; Chen, J.; Cui, B.; Yin, P.; Zhang, C. Design and Preparation of Biomass-Derived Carbon Materials for Supercapacitors: A Review. C 2018, 4, 53. [CrossRef]

6. Bedia, J.; Peñas-Garzón, M.; Gómez-Avilés, A.; Rodriguez, J.J.; Belver, C. A Review on the Synthesis and Characterization of Biomass-Derived Carbons for Adsorption of Emerging Contaminants from Water. $C$ 2018, 4, 63. [CrossRef]

(C) 2019 by the authors. Licensee MDPI, Basel, Switzerland. This article is an open access article distributed under the terms and conditions of the Creative Commons Attribution (CC BY) license (http://creativecommons.org/licenses/by/4.0/). 